Check for updates

Cite this: Chem. Sci., 2019, 10, 7375

๑ All publication charges for this article have been paid for by the Royal Society of Chemistry

Received 24th May 2019

Accepted 8th June 2019

DOI: $10.1039 / c 9 s c 02544 h$

rsc.li/chemical-science

\section{Mild synthesis of diboryldiborenes by diboration of $B-B$ triple bonds $\uparrow$}

\author{
Tobias Brückner, ${ }^{\text {ab }}$ Rian D. Dewhurst, (D) ab Theresa Dellermann, (D) ${ }^{\text {ab }}$ Marcel Müller ${ }^{\mathrm{ab}}$ \\ and Holger Braunschweig (DD *ab
}

A set of diboryldiborenes are prepared by the mild, catalyst-free, room-temperature diboration of the B-B triple bonds of doubly base-stabilized diborynes. Two of the product diboryldiborenes are found to be airand water-stable in the solid state, an effect that is attributed to their high crystallinity and extreme insolubility in a wide range of solvents.

\section{Introduction}

The propensity of polyboron species to form clusters as a way of quenching the natural electron deficiency of boron is now well documented. ${ }^{1}$ A consequence of this phenomenon is that networks of hypovalent boron atoms, bound through electronprecise $(2 \mathrm{e} 2 \mathrm{c}, 4 \mathrm{e} 2 \mathrm{c}$ or $6 \mathrm{e} 2 \mathrm{c})$ bonds, are extremely difficult to deliberately construct. ${ }^{2}$ Thus, a chemistry based on boron chains, analogous to the ubiquitous chain chemistry of carbon, is simply nonexistent. While boranes $\left(\mathrm{BR}_{3}\right)$ and diboranes(4) $\left(B_{2} R_{4}\right)^{3}$ are now relatively well studied compounds, even some of the most simple boron analogues of organic species, such as short chains and small cyclic species, are extremely rare and suffer from difficult syntheses. Nöth's syntheses of linear tri-, tetra-, penta- and hexaboranes in 1970 and $1994,{ }^{4}$ which are based on the reductive coupling of haloboranes, still represent some of the only rational synthetic routes to boron chain species, as exemplified in Fig. 1A. However, these synthetic routes rely on somewhat temperamental B-B coupling steps under harsh, functional-group-intolerant reductive conditions, making these reactions likely only possible with diorganylamino-substituted borane precursors. Nöth's boron chains have recently been supplemented by syntheses of $B_{4}$ chains using low-valent boron precursors. In 2012 we reported the unexpected transition-metal-templated catenation of four borylene ligands into a $\mathrm{B}_{4}$ chain (Fig. 1B)..$^{5}$ In the absence of transition metal templation, Kinjo and coworkers found that diboration of a geminally-base-stabilized $B_{2}$ species with

${ }^{a}$ Institut für Anorganische Chemie, Julius-Maximilians-Universität Würzburg, Am Hubland, 97074 Würzburg, Germany. E-mail: h.braunschweig@uni-wuerzburg.de

${ }^{b}$ Institute for Sustainable Chemistry \& Catalysis with Boron, Julius-MaximiliansUniversität Würzburg, Am Hubland, 97074 Würzburg, Germany

$\dagger$ Electronic supplementary information (ESI) available: Experimental details and NMR spectra. CCDC 1882019-1882023. For ESI and crystallographic data in CIF or other electronic format see DOI: $10.1039 / \mathrm{c} 9 \mathrm{sc} 02544 \mathrm{~h}$ bis(catecholato)diboron $\left(\mathrm{B}_{2}\right.$ cat $\left._{2}\right)$ provided a highly unusual $\mathrm{B}_{4}$ chain (Fig. 1C), ${ }^{6}$ while in our laboratories, the same reagent led to both 1,1- and 1,2-diborations of doubly base-stabilized diborenes $\left(\mathrm{LRB}=\mathrm{BRL}\right.$ ) (Fig. 1D). ${ }^{\text {? }}$

Another goal of short boron chain chemistry is the synthesis of chains with partially filled $\pi$ orbitals. In an attempt to prepare such species, in joint work with the groups of Vargas and Ingleson, we recently reported the reductive coupling of boryl-substituted dihaloborane Lewis adducts to form diboryldiborenes featuring significantly conjugated $\mathrm{B}_{4}$ chains with two $\pi$ electrons (Fig. 1E). ${ }^{8}$ These reactions, while allowing access to these unusual compounds, were hampered by the combination of the relatively labile B-B bond in the precursor and the harsh reductive conditions required. A more convergent and potentially advantageous strategy to prepare diborylated diborenes - as well as other difunctionalized diborenes - would be the direct addition of $\mathrm{E}-\mathrm{E} \sigma$ bonds across the $\mathrm{B} \equiv \mathrm{B}$ triple bond of diborynes (Fig. 1F). ${ }^{2}$ This strategy would eliminate the need for installing various functional groups directly into the Lewis-base-bound dihaloborane precursor $(\mathrm{LB}) \mathrm{BX}_{2}\left(\mathrm{ER}_{n}\right)(\mathrm{LB}=$ Lewis base; $\mathrm{ER}_{n}$ = functional group), as well as circumventing the incompatibility of this functional group with the harsh reduction conditions needed to form the $\mathrm{B}=\mathrm{B}$ double bond.

The success of such a strategy would require reliable 1,2addition chemistry of $\sigma$-bound $\mathrm{E}-\mathrm{E}$ species across the $\mathrm{B} \equiv \mathrm{B}$ triple bonds. While both doubly base-stabilized diborenes $(\mathrm{LRB}=\mathrm{BRL})$ and diborynes $(\mathrm{LB} \equiv \mathrm{BL})$ have shown diverse reactivity with unsaturated species and elemental chalcogens, ${ }^{2}$ they have shown only limited propensity to undergo simple 1,2addition reactions with other $\sigma$-bonded species, and often react unpredictably or not at all. In addition to the diboration reactions described above (Fig. 1D), doubly base-stabilized diborenes undergo hydroboration with the hydroborane HBCat; however, application of the more Lewis acidic borane 9-borabicyclo[3.3.1]nonane led instead to disproportionation and 


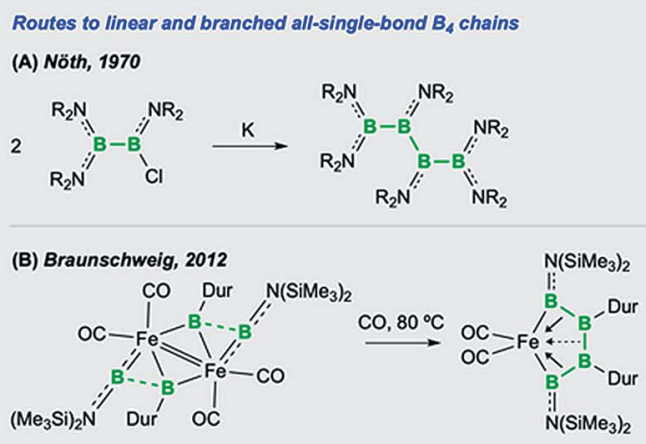

(C) Kinjo, 2018

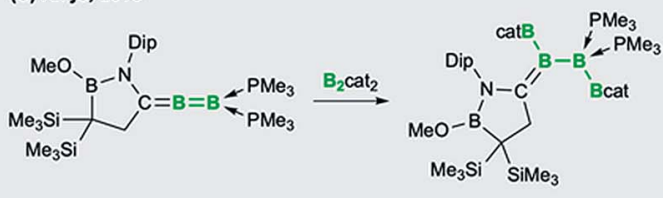

(D) Braunschweig, 2018
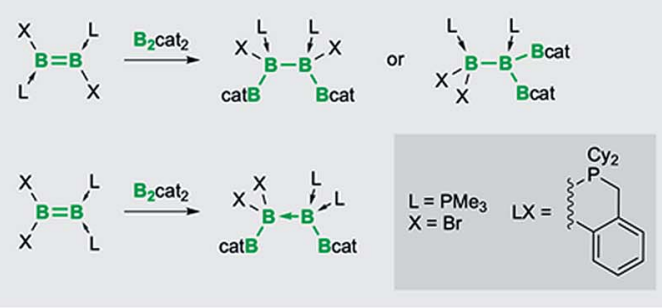

Routes to monounsaturated $B_{4}$ chains

(E) Vargas, Ingleson \& Braunschweig, 2018

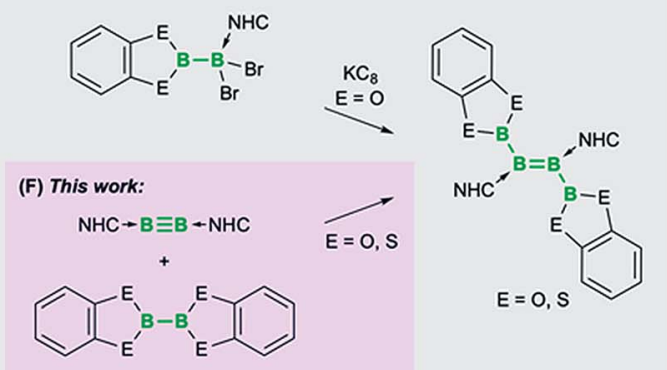

Fig. 1 Synthetic routes to saturated $(A-D)$ and monounsaturated ( $E$ and F) $\mathrm{B}_{4}$ chains ( $\mathrm{NHC}=\mathrm{N}$-heterocyclic carbene).

cluster formation. ${ }^{9}$ The only other confirmed 1,2-addition to a diborene consisted of an unexpected intramolecular $\mathrm{C}-\mathrm{H}$ addition across the $\mathrm{B}=\mathrm{B}$ bond. ${ }^{\mathbf{1 0}}$ Like diborenes, diborynes have also shown a marked reluctance to undergo conventional 1,2 -additions with labile $\sigma$-bonded species. Hydrogenation of diborynes has been demonstrated with some diborynes but appears not to take place with others. ${ }^{\mathbf{1 1}}$ In 2016 we reported that combination of diaryl ditellurides ArTeTeAr $\left(\mathrm{Ar}=\mathrm{Ph}, 4-\mathrm{C}_{6} \mathrm{H}_{4} \mathrm{~F}\right)$ to a diboryne resulted in addition of only one "TeAr" fragment to the $\mathrm{B}_{2}$ unit and formation of an aryltelluride salt. ${ }^{\mathbf{1 2}}$ However, the lighter diorganyldichalogenides were more recently found to undergo conventional 1,2-additions across diborynes, producing either diborenes or diradical products. ${ }^{13}$
We present herein convergent syntheses of monounsaturated $\mathrm{B}_{4}$ chains, doubly base-stabilized diboryldiborenes, by simple, uncatalyzed, room-temperature diboration of boronboron triple bonds. The products feature linear chains of four $\mathrm{sp}^{2}$-hybridized boron atoms, with the outer boron atoms possessing varying degrees of coplanarity and conjugation with the central $\mathrm{B}=\mathrm{B}$ double bonds.

\section{Results and discussion}

Combination of the doubly NHC-stabilized diboryne 1a (Fig. 2) with equimolar amounts of either $\mathrm{B}_{2} \mathrm{cat}_{2}$ or bis(dithiocatecholato)diboron $\left(\mathrm{B}_{2} \mathrm{Scat}_{2}\right)$, and stirring in benzene, led to a color change to blue and the emergence of pairs of new ${ }^{11} \mathrm{~B}$ NMR spectral signals. Drying of the solution and washing with hexane provided blue solids 2a,b (Fig. 2), which displayed ${ }^{11} \mathrm{~B}$ NMR signals (2a: $\delta$ 43.0, 27.7; 2b: $\delta$ 69.1, 29.6) differing from those of the precursors 1a $(\delta 56), \mathrm{B}_{2} \mathrm{cat}_{2}(\delta 31)$, and $\mathrm{B}_{2} \mathrm{Scat}_{2}(\delta 59)$. The upfield ${ }^{11} \mathrm{~B}$ NMR signals of $\mathbf{2 a}, \mathbf{b}$ fall at the lower-field end of those of known NHC-stabilized diborenes $(\delta 18-30),{ }^{2,13 a}$ and the pair of signals for $\mathbf{2 a}$ match those of the previously-reported diboryldiborenes bearing Bcat groups ( $\delta$ 42-44 and 27-28). ${ }^{8}$ The observation of single ${ }^{1} \mathrm{H}$ and ${ }^{13} \mathrm{C}$ NMR spectral resonances for the $\mathrm{CH}_{3}$ groups of the NHC donors indicated the symmetry of the molecule in solution. High-resolution LIFDI mass spectrometry confirmed the molecular formulae of $\mathbf{2 a , b}$ corresponding to the $1: 1$ addition of the precursor diborane to diboryne 1a.

The combination of diboryne $\mathbf{1} \mathbf{b}$, featuring unsymmetrically substituted NHC donors, with either $\mathrm{B}_{2}$ cat $_{2}$ or $\mathrm{B}_{2} \mathrm{Scat}_{2}$ led to a color change from red to brown and the precipitation of orange and red crystals, respectively (2c,d; Fig. 2). Highresolution LIFDI mass spectrometry and elemental analysis performed on these crystals again indicated $1: 1$ addition of the diborane to $\mathbf{1 b}$. The crystals of $\mathbf{2 c}, \mathbf{d}$ proved to be highly insoluble, allowing only partial characterization of $2 \mathrm{c}$ by solution ${ }^{1} \mathrm{H}$ and ${ }^{11} \mathrm{~B}$ NMR spectroscopy and precluding solution NMR spectroscopy for 2d. Diborene $2 \mathrm{c}$ showed a broad ${ }^{11} \mathrm{~B}$ NMR signal at $\delta 26$, but the remaining signal could not be identified
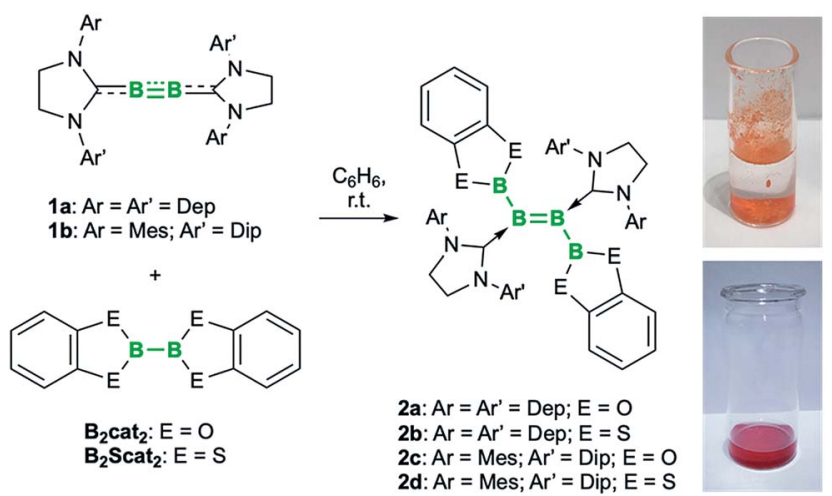

Fig. 2 Catalyst-free diboration of diborynes. Inset photos: suspensions of diborenes $2 \mathrm{c}$ (top) and $2 \mathrm{~d}$ (bottom) in water under ambient atmosphere. Dip =2,6-diisopropyl-phenyl; Dep =2,6-diethylphenyl. 

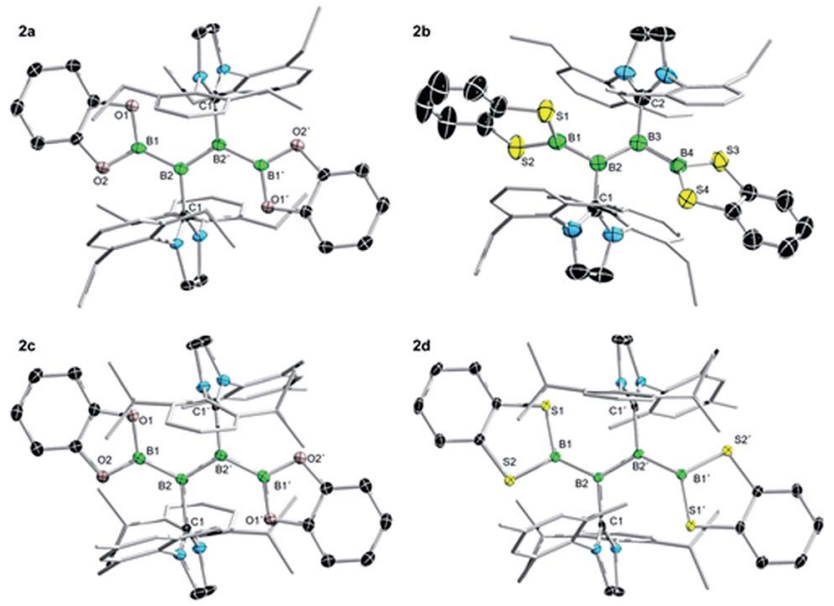

Fig. 3 Crystallographically derived structures of $2 a-d$. Ellipsoids shown at the $50 \%$ probability level. Some ellipsoids and all hydrogen atoms and solvent molecules (one molecule of $\mathrm{C}_{6} \mathrm{H}_{6}$ in $2 \mathrm{c}$ ) have been removed for clarity. Selected bond lengths $(\AA)$ and angles $\left(^{\circ}\right)$ for $2 a$ : B1B2 1.650(3), B2-B2' 1.605(3), O1-B1-B2-B2' 15.9(3). For 2b (two molecules in unit cell): B1-B2 1.685(4), 1.691(4), B2-B3 1.617(3), 1.619(3), B3-B4 1.689(4), 1.687(4), S1-B1-B2-B3 48.9(3), 52.9(3), S3B4-B3-B2 53.6(3), 50.3(3). For 2c: B1-B2 1.652(2), B2-B2' 1.608(3), O1-B1-B2-B2' 19.4(2). For 2d: B1-B2 1.664(2), B2-B2' 1.627(2), O1$\mathrm{B} 1-\mathrm{B} 2-\mathrm{B} 2^{\prime}$ 29.8(2).

due to the low concentration of the sample. Unfortunately, attempts to record solid-state MAS NMR spectra of 2 d provided either no signal (without rotation) or resulted in decomposition of the sample under the pressure created by the sample rotation.

Single-crystal X-ray diffraction analysis of $2 \mathbf{a}-\mathbf{d}$ provided final confirmation of the structures of the products (Fig. 3). While all four compounds show relatively long $\mathrm{B}=\mathrm{B}$ double bonds (2a:

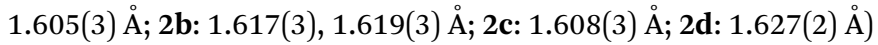
that could be indicative of increased $\pi$ conjugation with the outer boron atoms, the Bcat and BScat groups show a relatively broad range of $\mathrm{E}-\mathrm{B}-\mathrm{B}=\mathrm{B}$ torsion angles $\left(16-54^{\circ}\right)$, the larger of which indicate strong non-coplanarity and thus discount the presence of significant $\pi$ conjugation in the solid state. The outer B-B bond distances of 2a-d (2a: 1.650(3) Å; 2b: 1.685(4),

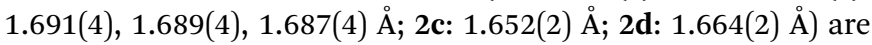
in line with those of the previously-reported diboryldiborenes (1.65-1.68 ̊).

We were surprised to observe that while the SIDepsubstituted diborenes $\mathbf{2 a , b}$ decompose within 15 minutes in air, diborenes $\mathbf{2 c , d}$ are stable for days in the solid state in ambient air and even as a suspension in water (Fig. 2, inset photos). Although the appearance of solid $2 \mathrm{c}$ remains unchanged, a small boronic acid signal can be observed in the ${ }^{1} \mathrm{H}$ and ${ }^{11} \mathrm{~B}$ NMR spectra after approximately one week in $\mathrm{D}_{2} \mathrm{O}$. These suspensions gradually decolorize within one hour upon addition of $\mathrm{CH}_{2} \mathrm{Cl}_{2}$, into which the diborenes are slowly solubilized and decomposed. As diborenes $\mathbf{2 a - d}$ are relatively similar in terms of electronics and sterics, the remarkable solidstate stability of $\mathbf{2 c , d}$ is likely a consequence of their high crystallinity. Once dissolved, even in miniscule amounts, the compounds quickly decompose in the presence of moisture.
The UV-vis spectra of the diboryldiborenes $\mathbf{2}$ are remarkably different in their features (see ESI $\dagger$ ). The spectrum of the orange compound 2c $(\lambda 451 \mathrm{~nm})$ resembles that of the previouslyreported yellow diboryldiborene ${ }^{8}[(\mathrm{IMe})(\mathrm{catB}) \mathrm{B}=\mathrm{B}(\mathrm{Bcat})(\mathrm{IMe})]$ (IMe $=1,3$-dimethylimidazol-2-ylidene; $\lambda_{\max }=435 \mathrm{~nm}$ ), both having low-wavelength features and no absorption in the longer wavelength region. However, the other three diborenes $\mathbf{2 a , b}, \mathbf{d}$ have significant absorptions in the region 550-650 $\mathrm{nm}(2 \mathrm{a}: \lambda$ 422, 578 (max.) nm; 2b: $\lambda$ 503, 608 (max.) nm; 2d: $\lambda 543$ (max.), $622 \mathrm{~nm})$. Overall the longer wavelength absorptions of 2ad relative to those of $[(\mathrm{IMe})(\mathrm{catB}) \mathrm{B}=\mathrm{B}$ (Bcat)(IMe)] suggest that the more $\sigma$-donating and $\pi$-withdrawing saturated-backbone NHCs in the former lead to significant decreases in the HOMO-LUMO gaps of the molecules.

It should also be noted that, in an attempt to induce double diboration, the diborynes $\mathbf{1 a}, \mathbf{b}$ were treated with two molar equivalents of the diboranes $\mathrm{B}_{2} \mathrm{cat}_{2}$ and $\mathrm{B}_{2} \mathrm{Scat}_{2}$. However, after monitoring conversion to the respective diboryldiborenes $\mathbf{2 a}, \mathbf{b}$, no further reaction was observed, even with heating $\left(100^{\circ} \mathrm{C}\right)$ or under photolytic conditions.

\section{Conclusions}

The diboryne diboration reactions herein provide convergent access to monounsaturated boron chains and provide a new tool in the challenging construction of electron-precise $\mathrm{B}-\mathrm{B}$ bonds. Moreover, the high stability of the bulky diboryldiborene products is very encouraging. The extreme sensitivity generally shown by diborenes is the main practical impediment towards their use as " $\pi$ superdonor" units in molecular electronic materials, thus the discovery of derivatives able to withstand air and water - even if only in the solid state - is a significant step forward.

\section{Conflicts of interest}

There are no conflicts to declare.

\section{Acknowledgements}

Financial support for this project was provided by the European Research Council (ERC) under the European Union Horizon 2020 Research and Innovation Program (Advanced Grant agreement no. 669054). The authors also thank Allychem Co., Ltd. (Dalian, China) for a generous gift of $\mathrm{B}_{2} \mathrm{cat}_{2}$ and Mr Dominic Prieschl for photography.

\section{Notes and references}

1 (a) A. Stock, Hydrides of Boron and Silicon, Cornell Univ. Press, Ithaca, NY, 1933; (b) W. N. Lipscomb, Adv. Inorg. Chem., 1959, 1, 117-156; (c) K. Wade, J. Chem. Soc., Chem. Commun., 1971, 792-793; (d) R. E. Williams, Inorg. Chem., 1971, 10, 210-214; (e) M. A. Fox and K. Wade, Pure Appl. Chem., 2003, 75, 1315-1323; $(f)$ E. Osorio, J. K. Olson, W. Tiznado and A. I. Boldyrev, Chem.-Eur. J., 2012, 18, 9677-9681. 
2 (a) K. Nozaki, Y. Aramaki, M. Yamashita, S.-H. Ueng, M. Malacria, E. Lacôte and D. P. Curran, J. Am. Chem. Soc., 2010, 132, 11449-11451; (b) H. Braunschweig and R. D. Dewhurst, Angew. Chem., Int. Ed., 2013, 52, 35743583; (c) M. Arrowsmith, H. Braunschweig and T. E. Stennett, Angew. Chem., Int. Ed., 2017, 56, 96-115.

3 (a) E. C. Neeve, S. J. Geier, I. A. I. Mkhalid, S. A. Westcott and T. B. Marder, Chem. Rev., 2016, 116, 9091-9161; (b) D. G. Hall, Boronic Acids: Preparation, Applications in Organic Synthesis and Medicine, Wiley-VCH, Weinheim, 2005.

4 (a) K. H. Hermannsdörfer, E. Matejčikova and H. Nöth, Chem. Ber./Recl., 1970, 103, 516-527; (b) G. Linti, D. Loderer, H. Nöth, K. Polborn and W. Rattay, Chem. Ber., 1994, 127, 1909-1922.

5 H. Braunschweig, Q. Ye, A. Vargas, R. D. Dewhurst, K. Radacki and A. Damme, Nat. Chem., 2012, 4, 563-567.

6 W. Lu, Y. Li and R. Kinjo, Angew. Chem., Int. Ed., 2018, 57, 15691-15695.

7 T. E. Stennett, R. Bertermann and H. Braunschweig, Angew. Chem., Int. Ed., 2018, 57, 15896-15901.

8 A. Hermann, J. Cid, J. D. Mattock, R. D. Dewhurst, I. Krummenacher, A. Vargas, M. J. Ingleson and
H. Braunschweig, Angew. Chem., Int. Ed., 2018, 57, 1009110095.

9 (a) H. Braunschweig, R. D. Dewhurst, C. Hörl, A. K. Phukan, F. Pinzner and S. Ullrich, Angew. Chem., Int. Ed., 2014, 53, 3241-3244; (b) H. Braunschweig and C. Hörl, Chem. Commun., 2014, 50, 10983-10985.

10 S. R. Wang, M. Arrowsmith, H. Braunschweig, R. D. Dewhurst, V. Paprocki and L. Winner, Chem. Commun., 2017, 53, 11945-11947.

11 M. Arrowsmith, J. Böhnke, H. Braunschweig, M. A. Celik, T. Dellermann and K. Hammond, Chem.-Eur. J., 2016, 22, 17169-17172.

12 H. Braunschweig, P. Constantinidis, T. Dellermann, W. C. Ewing, I. Fischer, M. Hess, F. R. Knight, A. Rempel, C. Schneider, S. Ullrich, A. Vargas and J. D. Woollins, Angew. Chem., Int. Ed., 2016, 55, 5606-5609.

13 (a) J. Böhnke, T. Dellermann, M. A. Celik, I. Krummenacher, R. D. Dewhurst, S. Demeshko, W. C. Ewing, K. Hammond, M. Heß, E. Bill, E. Welz, M. Röhr, R. Mitrić, B. Engels, F. Meyer and H. Braunschweig, Nat. Commun., 2018, 9, 1197; (b) E. Welz, J. Böhnke, R. D. Dewhurst, H. Braunschweig and B. Engels, J. Am. Chem. Soc., 2018, 140, 12580-12591. 